einstein

Official Publication of the Instituto Israelita

de Ensino e Pesquisa Albert Einstein

ISSN: 1679-4508 | e-ISSN: 2317-6385

\title{
Erratum: Microbiological findings of the maternal periodontitis associated to low birthweight
}

Erratum: Achados microbiológicos da periodontite materna associados ao baixo peso ao nascer

Mariana Cedraz de Oliveira', Isaac Suzart Gomes-Filho², Andreas Stöcker'1, Laerte Oliveira Barreto Neto², André do Nascimento Santos², Simone Seixas da Cruz ${ }^{3}$, Johelle Santana Passos-Soares', Michelle Miranda Lopes Falcão², José Roberto Cardoso Meireles², Gregory John Seymour ${ }^{4}$, Roberto Meyer ${ }^{1}$, Soraya Castro Trindade²

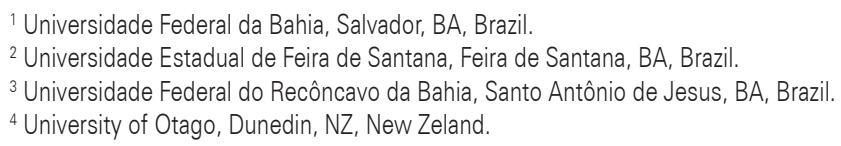

DOI: 10.31744/einstein_journal/2020A04209E

In the manuscript "Microbiological findings of the maternal periodontitis associated to low birthweight", DOI number: 10.31744/einstein_journal/ 2020AO4209, published at einstein (São Paulo). 2020;18:eAO4209, page 5, table 2:
Stated:
Treponema denticola ${ }^{\beta}$
Prevotella intermedia/ $/{ }^{\beta}$
Porphyromonas gingivalis ${ }^{\beta}$
Tannerella forsythia ${ }^{3}$
It should be read:
Porphyromonas gingivalis ${ }^{\beta}$
Treponema denticola ${ }^{\beta}$
Tannerella forsythia ${ }^{\beta}$
Prevotella intermedia ${ }^{\beta}$

\section{AUTHORS' INFORMATION}

Oliveira MC: http://orcid.org/0000-0003-0393-2420

Gomes-Filho IS: http://orcid.org/0000-0002-4270-8491

Stöcker A: http://orcid.org/0000-0001-8986-3133

Barreto Neto LO: http://orcid.org/0000-0001-9090-9063

Santos AN: http://orcid.org/0000-0002-2042-7410

Cruz SS: http://orcid.org/0000-0003-2828-0887

Passos-Soares JS: http://orcid.org/0000-0002-4541-1730

Falcão MM: http://orcid.org/0000-0002-0929-2324

Meireles JR: http://orcid.org/0000-0002-2008-4753

Seymour GJ: http://orcid.org/0000-0001-7595-5651

Meyer R: http://orcid.org/0000-0002-4727-4805

Trindade SC: http://orcid.org/0000-0001-7125-9114

\section{Copyright 2020}

